\title{
A case report of a patient presented with skin ulcer after treatment of lenvatinib
}

\author{
Serin Cha, Dong Woo Kim, Jung Wan Choe, Tae Hyung Kim, Seung Young Kim, Jong Jin Hyun, Sung Woo Jung, \\ Ja Seol Koo, Young Kul Jung, Hyung Joon Yim \\ Department of Internal Medicine, Korea University Ansan Hospital, Ansan, Korea
}

Received Jul. 20, 2021

Revised Sep. 18, 2021

Accepted Sep. 20, 2021
A 60-year-old man diagnosed with unresectable hepatocellular carcinoma $(\mathrm{HCC})$ presented to the hospital with pain in the perineal region. He had been taking lenvatinib every day for 2 months after he was diagnosed with HCC with metastases to the lymph node, small bowel mesentery, and retroperitoneal space. Enhanced abdominal computed tomography revealed mild elevation in intensity in the perineal subcutaneous tissue with subcutaneous emphysema. The patient was diagnosed with Common Terminology Criteria for Adverse Events grade 3, skin ulceration of stage IV with full-thickness skin loss and tissue necrosis in the muscular layer. The patient was taken off the medication with prescription of antibiotics, and after 3 weeks, the skin has fully recovered. This is the first report of an HCC patient who presented with a skin ulceration of stage IV after lenvatinib treatment. We recommend stopping the medication immediately and changing to alternative treatments with appropriate supportive care. (J Liver Cancer 2021;21:194-198)

Keywords: Lenvatinib mesylate; Skin ulcer; Hepatocellular carcinoma; Adverse drug event; Protein kinase inhibitor

\section{INTRODUCTION}

Lenvatinib is used as an alternative anti-cancer drug in patients with unresectable hepatocellular carcinoma (HCC) who have previously failed to primary anticancer therapy or sorafenib. ${ }^{1}$ Lenvatinib acts as an inhibitor of vascular endothelial growth factor receptors-1, 2, 3 and fibroblast growth factor receptors, ${ }^{2}$ and in the REFLECT study, patients diagnosed with unresectable liver cancer treated with lenvatinib showed non-inferiority to sorafenib, which lead to its ap-

\section{Corresponding author: Young Kul Jung}

Department of Internal Medicine, Korea University Ansan Hospital, 123 Jeokgeum-ro, Danwon-gu, Ansan 15355, Korea

Tel. +82-31-412-7623, Fax. +82-31-412-5582

E-mail: 93cool@korea.ac.kr proval by the Food and Drug Administration of the United States in 2018. ${ }^{3}$ In addition, according to a study by Liu et $\mathrm{al}^{4}$, combination of lenvatinib and pembrolizumab therapy showed superiority to sorafenib. However, lenvatinib is also known to cause side effects such as high blood pressure, diarrhea, fatigue, decreased appetite and weight, nausea, vomiting, and stomatitis. ${ }^{5}$ Occurrence of cutaneous ulcers after lenvatinib treatment is rare. ${ }^{6,7}$ Herein, we present a rare case of a patient who developed a cutaneous ulcer after lenvatinib treatment for an unresectable HCC.

This case report is described according to the CARE guidelines available from https://www.care-statement.org/. The ethics committee of Korea University Ansan Hospital approved of this study (IRB approval number: 2021AS0225), and waived the requirement for informed consent. 


\section{CASE REPORT}

A 60-year-old man visited the emergency department complaining of perineal pain and an ulcerative cutaneous lesion. Four months prior to this visit, he had visited the hospital with abdominal distension, and was diagnosed with chronic hepatitis B and HCC. He also had a history of alcohol abuse, drinking one bottle of soju every day for 20 years. He was never a smoker, and did not have any other medical or surgical history.

A three-phase computed tomography (CT) at that time demonstrated an HCC with metastases to the lymph nodes in the porta hepatis, small bowel mesentery and retroperitoneal space. Carcinoma peritonei was suspected, and portal vein thrombosis (PVT) was also present (Fig. 1). Primovist liver magnetic resonance imaging showed an infiltrative HCC with intrahepatic metastases in both hepatic lobes, with tumor thrombi in the main and right portal veins as well as the right hepatic vein. Due to multiple lymph node metastases in the porta hepatis with suspicious seeding nodules in the right lower abdominal cavity, the patient was diagnosed as HCC, Barcelona Clinic Liver Cancer stage C. Hence, he
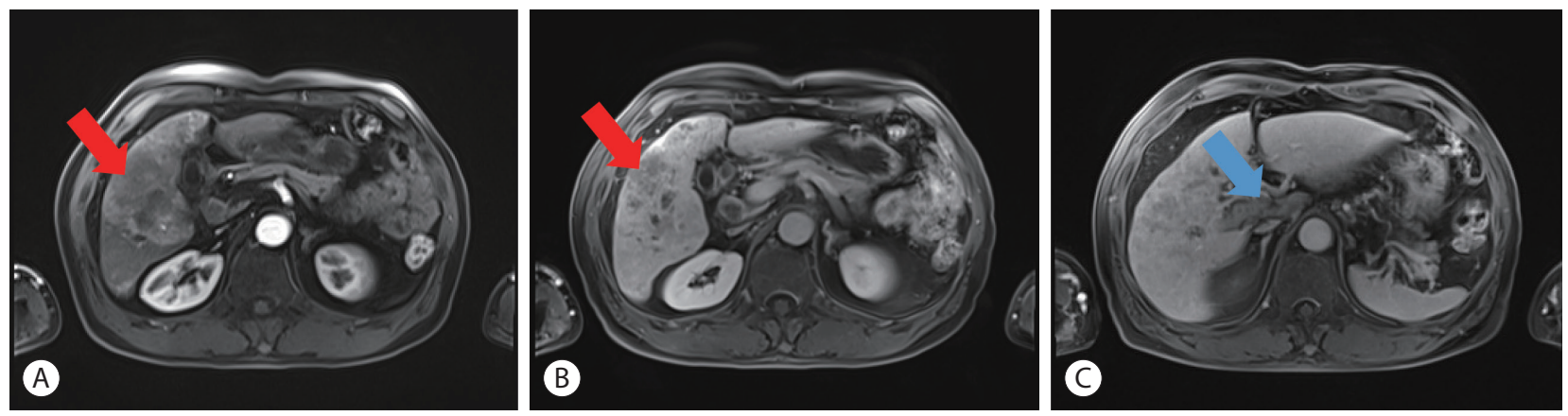

Figure 1. The primovist-enhanced magnetic resonance imaging of the hepatocellular carcinoma at initial diagnosis: (A) early enhancement of the tumor in the T1 arterial enhanced phase (red arrow), (B) early washout in venous phase (red arrow), and (C) a tumor thrombosis in the main portal vein in portal phase (blue arrow).
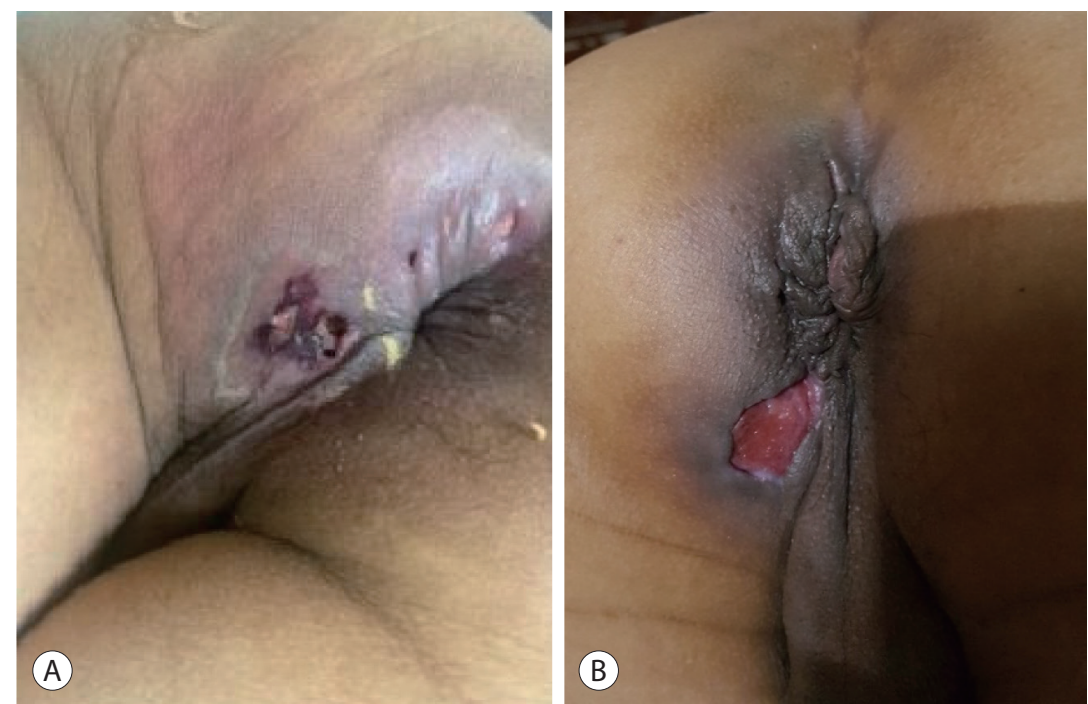

Figure 2. Photograph of the cutaneous lesion. (A) The buttock lesion at initial presentation after 2 months of Lenvatinib. Ulceration is observed in the perineal area, extending from behind the scrotum to the anus. The patient complained of perineal pain, and the skin lesion was associated with bloody discharge, full-thickness skin loss and tissue necrosis, corresponding to Common Terminology Criteria for Adverse Events grade 3, skin ulceration of stage IV. (B) The cutaneous lesion improved dramatically after 10 days after discontinuation of lenvatinib, surgical procedure and antibiotic treatment. 
was started on treatment with lenvatinib.

At the time of his second visit, he was on lenvatinib $12 \mathrm{mg}$ every day, with concurrent radiotherapy for the PVT. His body weight was $65.8 \mathrm{~kg}$, and his Eastern Cooperative Oncology Group status was 0 .

After taking lenvatinib for 2 months, he visited the emergency room complaining of pain in the perineal area. He seemed to be in acute pain with vital signs as follows: blood pressure of $150 / 104 \mathrm{mmHg}$, heart rate of $84 / \mathrm{min}$, respiratory rate of $16 / \mathrm{min}$, and body temperature of 36.9 . He was alert without icteric sclera. Chest auscultation revealed normal breath sounds and his heartbeat was regular without murmurs. On abdominal examination, his abdomen was soft without rigidity, and his liver and spleen were not palpable. Neurological examination was unremarkable. On examining the skin, an ulcerative skin lesion was observed in the perineal area, extending from behind the right scrotum to the anus (Fig. 2).

Initial laboratory findings were as follows: hemoglobin $16.6 \mathrm{~g} / \mathrm{dL}$, white blood cell count $5,800 / \mu \mathrm{L}$ (neutrophil $73.3 \%$, lymphocyte $14.3 \%$, monocyte $11.9 \%$, eosinophil $0.2 \%$, basophil $0.3 \%$ ), platelet count $188,000 / \mu \mathrm{L}$, blood urea nitrogen $30.2 \mathrm{mg} / \mathrm{dL}$, serum creatinine $1.25 \mathrm{mg} / \mathrm{dL}$, asparate aminotransferase $92 \mathrm{IU} / \mathrm{L}$, alanine aminotransferase $25 \mathrm{IU} / \mathrm{L}$, total bilirubin $1.89 \mathrm{mg} / \mathrm{dL}$, direct bilirubin $0.92 \mathrm{mg} / \mathrm{dL}$, total protein $2.8 \mathrm{~g} / \mathrm{dL}$, albumin $2.8 \mathrm{~g} / \mathrm{dL}$, and C-reactive protein (CRP) $9.93 \mathrm{mg} / \mathrm{dL}$.

Enhanced abdominal CT demonstrated no evidence of abscess, but high attenuation was observed in the subcutaneous tissue, which was interpreted as being most likely due to inflammation and subcutaneous emphysema caused by the ulcer. There was no significant difference in the size of the hepatic mass and the adjacent lymph node compared to the CT scan taken 2 months ago.

The patient was diagnosed with CTCAE grade 3, skin ulceration of stage IV due to lenvatinib treatment, with fullthickness skin loss and tissue necrosis. ${ }^{8}$ He immediately stopped taking lenvatinib, a wet gauge dressing was applied, and piperacillin/tazobactam was prescribed. He experienced severe pain, and on the third day, the laboratory examination showed an increase in CRP level to $13.95 \mathrm{mg} / \mathrm{dL}$. Therefore, on the fourth day, the patient underwent an incision of the necrotic tissue under local anesthesia. The largest skin ulcer measured about $4 \times 3 \mathrm{~cm}$ and no other complication was observed during the surgery. After 10 days of antibiotics and conservative treatment following the surgical incision (Fig. 2), the patient was discharged, with healing of the cutaneous lesion, amelioration of the pain, as well as a decrease in the CRP level to $5.05 \mathrm{mg} / \mathrm{dL}$. He was educated to self-disinfect the cutaneous lesion, and full recovery was noted after 3 weeks.

\section{DISCUSSION}

Lenvatinib is a type I tyrosine kinase inhibitor with antiangiogenic activity. ${ }^{9,10}$ Due to this property, lenvatinib is used to treat liver cancer, differentiated thyroid cancer and renal cell carcinoma, and there are recent studies that have also demonstrated its effectiveness on medullary and anaplastic thyroid cancer, as well as endometrial cancer. ${ }^{11,12}$

However, lenvatinib is also known for its various side effects, such as hypertension, diarrhea, nausea, vomiting and hypothyroidism. In addition to these side effects, cutaneous adverse effects have also been reported. ${ }^{5,13}$ Previous studies have reported complications including palmar-plantar erythrodysesthesia (27\% to $32 \%)$, skin rash (14\% to $21 \%$ ), alopecia $(12 \%)$, hyperkeratosis $(7 \%)$, and wound healing impairment $(<1 \%) .{ }^{14,15}$ However, there is a limited number of studies reporting skin ulceration as a side effect; a thorough search for documented case reports retrieved two case studies of ulcer formation after lenvatinib treatment. Kitamura et al. ${ }^{7}$ reported a case of a cutaneous ulcer that occurred in the subclavicular area of a patient with relapsed thyroid cancer after treatment with lenvatinib. Lenvatinib was immediately ceased and a pectoralis major myocutaneous flap was grafted over the ulcerated lesion. However, this case was different from our present case, as the cutaneous lesion occurred close to the site of the primary cancer. Dohmen ${ }^{6}$ reported a case of a palmar-plantar erythrodysesthesia in an HCC patient after lenvatinib treatment which recurred within 3 weeks after restarting the drug, after a 4-week-long withdrawal period. The recurrent palmar-plantar erythrodysesthesia was accompa- 
nied by an ulcerative cutaneous lesion in the thigh. The skin lesion, CTCAE grade 2, was erythematous with partial thickness loss in transitional stage to formation of a new skin. The lesion disappeared about 4 weeks after discontinuation of the medication and application of prostaglandin ointment. ${ }^{6}$ This case is quite similar to our case as the skin lesion was formed distant from the primary tumor; however, the skin lesion was not as severe as in our case. Our case is the first case of a cutaneous ulcer after lenvatinib treatment with a CTCAE grade of 3 , that was observed in a distant location from the primary cancer. The precise mechanism by which lenvatinib causes cutaneous ulceration is not fully understood, but platelet-derived growth factor receptor inhibition by lenvatinib may play a role in impairing the tissue repair process. ${ }^{8}$

The withdrawal period of lenvatinib and the treatment ulcer formation due to lenvatinib, has yet to be established. In the case reported by Kitamura et al. ${ }^{7}$, lenvatinib was ceased for 20 days and was restarted after the surgery; however, after 1 month of restarting lenvatinib, they stopped again due to fistula formation and discharge through the fistula. Similarly, in Dohmen's case report, the palmar-plantar erythrodysesthesia recurred with stage II ulcer on restarting the medication after a 4-week withdrawal period, and the medication had to be stopped again. ${ }^{6}$ Therefore, both case reports demonstrate that the cutaneous lesion recurred or worsened after restarting lenvatinib. In contrast, our case showed severe skin ulceration of stage IV even when the lenvatinib was not restarted. Therefore, close observation and immediate cessation of lenvatinib is warranted when a cutaneous ulcer is detected, and alternative medications should be strongly considered.

This is the first case report of an HCC patient who presented with skin ulceration of stage IV after treatment with lenvatinib. We recommend quitting the medication immediately and also changing to alternative treatment even when the lesion shows significant improvement after appropriate supportive measures.

\section{Conflicts of Interest}

The authors have no conflicts of interest to disclose.

\section{Ethics Statement}

The ethics committee of Korea University Ansan Hospital approved of this study (IRB approval number: 2021AS0225), and waived the requirement for informed consent.

\section{Funding Statement}

No funding to declare.

\section{Data Availability}

Data sharing not applicable to this article as no datasets were generated or analyzed for this case report.

\section{ORCID}

$\begin{array}{ll}\text { Serin Cha } & \text { https:/orcid.org/0000-0002-3863-4768 } \\ \text { Dong Woo Kim } & \text { https:/orcid.org/0000-0001-6767-426X } \\ \text { Jung Wan Choe } & \text { https:/orcid.org/0000-0003-0634-5141 } \\ \text { Tae Hyung Kim } & \text { https://orcid.org/0000-0002-7747-4293 } \\ \text { Seung Young Kim } & \text { https://orcid.org/0000-0001-8933-7715 } \\ \text { Jong Jin Hyun } & \text { https://orcid.org/0000-0002-5632-7091 } \\ \text { Sung Woo Jung } & \text { https://orcid.org/0000-0003-1954-1678 } \\ \text { Ja Seol Koo } & \text { https://orcid.org/0000-0002-1202-075X } \\ \text { Young Kul Jung } & \text { https://orcid.org/0000-0002-6566-1382 } \\ \text { Hyung Joon Yim } & \text { https://orcid.org/0000-0002-6036-2754 }\end{array}$

\section{Author Contribution}

\author{
Conceptualization: SC, THK, YKJ, HJY \\ Data curation: SC, DWK, SWJ, JJH, JSK \\ Methodology: JWC, THK, SYK, SWJ, YKJ \\ Project administration: DWK, JWC, SYK, YKJ, HJY \\ Writing-original draft: SC, JJH, JSK, HJY \\ Writing-review \& editing: SC, DWK, JWC, THK, SYK, JJH, \\ SWJ, JSK, YKJ, HJY
}

Approval of final manuscript: all authors.

\section{References}

1. Dipasquale A, Marinello A, Santoro A. A comparison of lenvatinib versus sorafenib in the first-line treatment of unresectable hepatocellular carcinoma: selection criteria to guide physician's choice in a new therapeutic scenario. J Hepatocell Carcinoma 2021;8:241251.

2. Kudo M, Finn RS, Qin S, Han KH, Ikeda K, Piscaglia F, et al. Len- 
vatinib versus sorafenib in first-line treatment of patients with unresectable hepatocellular carcinoma: a randomised phase 3 noninferiority trial. Lancet 2018;391:1163-1173.

3. Yamashita T, Kudo M, Ikeda K, Izumi N, Tateishi R, Ikeda M, et al. REFLECT-a phase 3 trial comparing efficacy and safety of lenvatinib to sorafenib for the treatment of unresectable hepatocellular carcinoma: an analysis of Japanese subset. J Gastroenterol 2020;55:113-122.

4. Liu Z, Li X, He X, Xu Y, Wang X. Complete response to the combination of Lenvatinib and Pembrolizumab in an advanced hepatocellular carcinoma patient: a case report. BMC Cancer 2019;19:1062.

5. European Medicines Agency. Lenvima. 2015 [Internet]. Amsterdam (NL): European Medicines Agency; [cited 2015 Mar 26]. Available from: https://www.ema.europa.eu/en/documents/assessmentreport/lenvima-epar-public-assessment-report_en.pdf.

6. Dohmen K. Severe ulcerative skin lesions due to lenvatinib. Clin Gastroenterol Hepatol 2020;18:e113.

7. Kitamura M, Hayashi T, Suzuki C, Hirano S, Tateya I, Kishimoto $Y$, et al. Successful recovery from a subclavicular ulcer caused by lenvatinib for thyroid cancer: a case report. World I Surg Oncol 2017;15:24.

8. U.S. Department of Health and Human Services. Common Terminology Criteria Adverse Events (CTCAE) Version 4.0, 2009 [Internet]. Washington, D.C. (USA): U.S. Department of Health and Human Services; [cited 2009 May 28]. Available from: https://evs.nci.nih.gov/ftp1/CTCAE/CTCAE_4.03/Archive/CT-
CAE_4.0_2009-05-29_QuickReference_8.5x11.pdf.

9. Yamamoto Y, Matsui J, Matsushima T, Obaishi H, Miyazaki K, Nakamura $K$, et al. Lenvatinib, an angiogenesis inhibitor targeting VEGFR/FGFR, shows broad antitumor activity in human tumor xenograft models associated with microvessel density and pericyte coverage. Vasc Cell 2014;6:18.

10. Schlumberger $M$, Jarzab $B$, Cabanillas ME, Robinson B, Pacini F, Ball DW, et al. A phase II trial of the multitargeted tyrosine kinase inhibitor lenvatinib (E7080) in advanced medullary thyroid cancer. Clin Cancer Res 2016;22:44-53.

11. Tahara M, Kiyota N, Yamazaki T, Chayahara N, Nakano K, Inagaki $L$, et al. Lenvatinib for anaplastic thyroid cancer. Front Oncol 2017;7:25.

12. Fleeman N, Houten R, Chaplin M, Beale S, Boland A, Dundar Y, et al. A systematic review of lenvatinib and sorafenib for treating progressive, locally advanced or metastatic, differentiated thyroid cancer after treatment with radioactive iodine. BMC Cancer 2019;19:1209.

13. Suyama K, Iwase H. Lenvatinib: a promising molecular targeted agent for multiple cancers. Cancer Control 2018;25:1073274818789361.

14. Takahashi S, Kiyota N, Tahara M. Optimal use of lenvatinib in the treatment of advanced thyroid cancer. Cancers Head Neck 2017;2:7.

15. Kim SY, Kim SM, Chang H, Kim BW, Lee YS, Chang HS, et al. Safety of tyrosine kinase inhibitors in patients with differentiated thyroid cancer: real-world use of lenvatinib and sorafenib in Korea. Front Endocrinol 2019;10:384. 